\title{
Employing New Mathematical Models and Equations to Evaluate Risk-Benefit Criteria of Clinical Therapeutics
}

\author{
${ }^{1}$ Da Yong Lu, ${ }^{2}$ Ting Ren Lu and ${ }^{3}$ Jian Ding \\ ${ }^{1}$ School of Life Sciences, Shanghai University, Shanghai 200444, PR China \\ ${ }^{2}$ College of Sciences, Shanghai University, Shanghai 200444, PR China \\ ${ }^{3}$ Shanghai Institute of Materia, Chinese Academy of Sciences 201203, PR China
}

\begin{abstract}
Current preclinical and clinical evaluation of a drugs or therapy is at first to find out a maximum toxicity tolerance, which is overall fixed. The potential therapeutic dosage will be ranged just lower or within the periphery of these maximum tolerant data. A hidden acceptance among general researchers lies as the toxicity concentration of a drug is always the same and can be referred as a fixed data. However, practical therapeutics is not very strictly following this doctrine. Even using many strict well-formed mathematical models and toxicity evaluating systems, the disputes and lawsuits of some newly-developed drugs are increasing dramatically nowadays. In this work, a mathematical equation and a dynamic parameter $\tau$ are generated to help improving this situation. This new mathematic model combines and integrates effective, toxicity and no effective data as a whole, which fits to evaluate the risk-benefits of therapeutics in dynamic and changeable states. We need no more to deduce therapeutic dosage from fixed tolerance data regardless different therapeutic modes of action and toxicity of drugs acting on different organs and physiological systems. We hypothesize and equation that risk-benefit ratios are varied with drug dosages. It is a new start to help the understanding of effects and toxicities of therapeutic same time in a single clinical practice and well compliment with previous mathematical models.
\end{abstract}

Key words: Mathematical models, risk-benefit criteria, clinical therapeutics

\section{INTROCUCTION}

Current preclinical and clinical evaluation of a drugs or therapy is at first to find out a maximum toxicity tolerance, which is overall fixed. The potential therapeutic dosage to test will be ranged just lower or within the periphery of these maximum tolerant data - they are in a very narrow dose ranges. A hidden acceptance among general researchers lies as the toxicity concentration of a drug is always the same and can be referred as a fixed data. However, practical therapeutics is not very strictly following this doctrine. Even using many strict routine mathematical models and toxicity evaluating systems, the disputes and lawsuit of some new developed drugs which need wider-ranges of therapeutic dosage to take effective or test are increasing dramatically. With the increasing number of clinical therapeutic dispute, especially to newly-developed drugs such as anti-depressant ${ }^{[1,2]}$ and anticancer therapy ${ }^{[3]}$, call for renewed study for them is also mounting. To the general characteristics of these drugs, there is always a phenomenon that rising of drug dosages in patients will dramatically improve the symptoms of the diseases meanwhile their risks of impairment to normal physiological conditions and unknown drug-induced pathogenesis are also enlarged. However, the doses of these drugs in preclinical and clinical studies are not parallel between therapeutic effects and toxicity. It is often neglect their clinical outcomes in low dosages. Clinicians, in many circumstances, often face with ambiguity to some novelty of therapeutics because many of their attempts will lead to controversial and disputes. We herein design a mathematical equation to help improving the situations by using more rigorous and comprehensive surveillance systems for drugs in patients.

Problems and new hypotheses: Currently norm of pre-clinical drug investigations are to suppose there is parallel between lethal or tolerance dosage in animals or in humans with effects and benefit outcomes in all drugs dosage attempted. Drug maximum tolerance dosages are often fixed and become a limitation for obtaining sufficient data of drug effective evaluation in relative higher or lower dose-range by allowing narrow ranges of tested doses of a therapy.

Our hypotheses are based on; (i) we must not limit tests of effective and toxicity dosage from a fixed lethal or tolerance dosages in all circumstances; (ii) we propose that the effective-toxicity ratio may be varied 
with drug dosage used, it can be represented in a dynamic way; (iii) the toxicity of drugs to different organs and physiological systems could be calculated as a whole. To address this problem, a new variable parameter $(\tau)$ and a relevant mathematical equation are generated to represent the biological significance and effectiveness by $\tau$ from variables of effective-toxicity-no response. It will help the drug developers and drug surveillance institution to better understand the status of drugs in patients before a drug is finally manufactured.

The equation is given as follow:

$\tau_{\text {conc }}=\frac{\sum\left(R_{E} \times p_{E}\right)-l \sum\left(R_{T} \times p_{T}\right)}{P_{n o}}$

Here denote the parameters of all variables in the equation.

$R_{E}$ and $R_{T}$ represent the percentage rate of effectiveness and toxicity respectively (they will be counted as $10 \%, 20 \% \cdots 90 \%$ or $100 \%$ and further multiple them with their probability $\left(P_{E}\right.$ or $\left.P_{T}\right)$ being occurred or measured. $L$ represents different possibility of toxicity to occur, such as renal, cardio-vascular. $P_{\text {no }}$ means the data of probability of no any effective or toxicity phenomena.

Further explanations for their biological significances: For the sign $\tau$, the highest $\tau$ for a therapy may be sought and use. $\tau$ is a dynamic data and could be a negative data in some harmful conditions and $\tau_{0}$ must be $0 . \tau_{\text {conc }}$ could represent as either drug concentrations in patient's blood or dosage of drugs given to a patient.

\section{CONCLUSION}

Our idea and solution to tackle the problems of evaluating therapeutic outcomes is to combine effective-toxicity-non-effective data in a single parameter and equation. More importantly, we hypothesize that the risk-benefits ratio of a therapy are correlated with the doses of the therapy. Its significance is to provide a new way of evaluating drugs presence in humans and can be used to currently used mathematical means and be applied in implementing individualized therapy ${ }^{[4]}$.

Overall, it might be mathematical models somewhat better than present ones and is the beginning for introducing different kinds of mathematical options in observation of risk-benefits relations of new drugs and therapeutic protocols in rapidity while the other routine methods can still be used. It is a novel attempt to integrate more information open for further discussions and researches.

\section{REFERENCES}

1. Check, E., 2004. Drug suicide risks prompt call for FDA action. Nature, 427: 474.

2. Holden, C., 2004. FDA weighs suicide risk in children on antidepressants. Science, 303: 745.

3. Cavazzana-Calvo, M., A. Thrasher and F. Mavilio, 2004. The future of gene therapy, balancing the risks and benefits of clinical trials. Nature, 427: 779-781.

4. Lu, D.Y., X.L. Chen and J. Ding, 2006. Individualized cancer chemotherapy, integrating drug sensitivity tests, pathological profile analysis and computational coordination - an effective strategy to improve clinical treatment. Med. Hypotheses, 66: 45-51. 